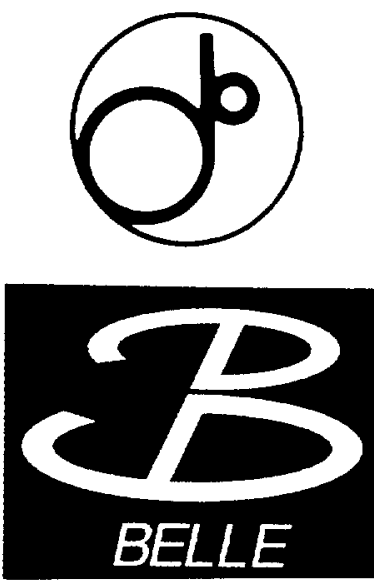

KEK Preprint 96-172

BELLE Preprint 97-03

OULNS 96-06

February 1997

H

\title{
Measurement of the Spatial Resolution of Wide-pitch Silicon Strip Detectors with Large Incident Angle*
}

T.Kawasaki ${ }^{a \dagger}$, M.Hazumi $^{a}$, Y.Nagashima ${ }^{a}$, K.Senyo $^{a}$, K.Sumisawa ${ }^{a}$, T.Takegai ${ }^{a}$, J.Haba ${ }^{b}$, T.Matsuda ${ }^{b}$, H.Ozaki $^{b}$, T.Tsuboyama ${ }^{b}$

${ }^{a}$ Graduate School of Science, Osaka University, Toyonaka 560, Japan

${ }^{b}$ Physics Department, National Laboratory for High Energy Physics, Tsukuba 305, Japan

*submitted to IEEE Transaction.

†Internet address: kawasaki@champ.hep.sci.osaka-y.ac.jp

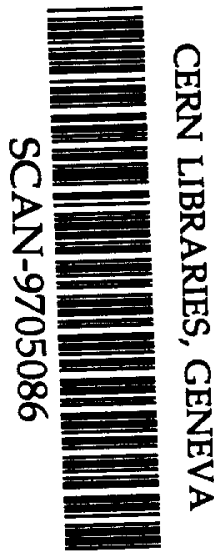


National Laboratory for High Energy Physics, 1997

KEK Reports are available from:

Technical Information \& Library

National Laboratory for High Energy Physics

1-1 Oho, Tsukuba-shi

Ibaraki-ken, 305

JAPAN

Phone: 0298-64-5136

Telex: 3652-534 (Domestic)

(0)3652-534 (International)

Fax: $\quad$ 0298-64-4604

Cable: $\quad$ KEK OHO

E-mail: Library@kekvax.kek.jp

Internet: http://www.kek.jp 


\section{Abstract}

As a part of R\&D for the BELLE experiment at KEK-B[1], we measured the spatial resolution of silicon strip detectors for particles with incident angles ranging from $0^{\circ}$ to $75^{\circ}$. These detectors have strips with pitches of 50,125 and $250 \mu \mathrm{m}$ on the ohmic side. We have obtained the incident angle dependence which agreed well with a Monte Carlo simulation. The resolution was found to be $11 \mu \mathrm{m}$ for normal incidence with a pitch of $50 \mu \mathrm{m}$, and $29 \mu \mathrm{m}$ for incident angle of $75^{\circ}$ with a pitch of $250 \mu \mathrm{m}$. 


\section{INTRODUCTION}

Nowadays the silicon micro strip detector is widely used for precise vertex measurement in particle physics at colliders. In the design of such vertex detectors, the size of detectors is required to be large in order to achieve the large acceptance. The large size of detector increases the number of readout channels and detector capacitance. The increase of noise because of larger capacitance is a serious problem.

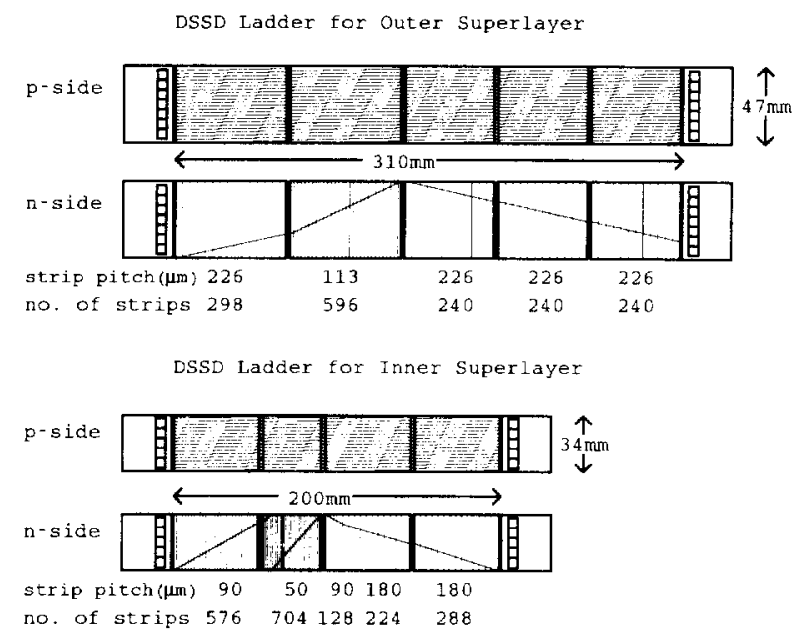

Fig. 1: The Double-Sided Silicon Detectors (DSSDs) for BELLE SVD. The strip pitches on the n-side (for Z coordinate measurement) are shown in the figure.

It is one of solutions to use detectors with the wide pitch at a region for a large incident angle where the position resolution is deteriorated by multiple scattering in a large amount of material crossed by the particles. At the BELLE experiment, particles penetrate the silicon vertex detector(SVD) with some incident angle because of its cylindrical configuration[2]. For $\mathrm{n}^{+}$-strips the maximum incident angle is $73^{\circ}$. In the design of BELLE-SVD, for reduction of the number of readout strips and capacitance, pitches of $\mathrm{n}^{+}$-strips are ranging from 50 to $226 \mu \mathrm{m}$ (Fig.1). To establish the design of the silicon micro strip detector with the wide strip pitch, it is necessary to check that there is no sizable loss of charge collection efficiency. In a wide pitch detector, there is a region of weak electric field in the middle of $\mathrm{n}^{+}$strips where efficiency of charge collection might be lower. With a large incident angle, therefore, the existence of low efficiency region might affect the spatial resolution. While there are studies on the wide-pitch detectors on the junction side[3], it has not been demonstrated that wide pitch $\mathrm{n}^{+}$-strip detectors on $\mathrm{n}$-bulk give a good spatial resolution for large incident angles.

In this test, we measured the spatial resolution of SSDs on the condition that the strip pitch on the ohmic side ranges from 50 to $250 \mu \mathrm{m}$ and the incident angle from $0^{\circ}$ to $75^{\circ}$.

\section{Measurement}

\section{A. Testbeam setup}

The beamtest was performed at the $\pi 2$ beam line at KEK(National Laboratory for High Energy Physics) $12 \mathrm{GeV}$ Proton Synchrotron in November 1995. During all of the data taking period, we used a $\pi^{-}$beam with momentum of $2.0 \mathrm{GeV} / \mathrm{c}$. Fig. 2 shows our testbeam setup. Four reference silicon strip detectors(SSD) are located upstream and downstream to reconstruct a particle track precisely. These SSDs are single-sided and have an area of $12.8 \times 64.0 \mathrm{~mm}^{2}$. The $\mathrm{p}^{+}$-strip pitch is $25 \mu \mathrm{m}$ and we read out every other strip, i.e. the readout pitch is $50 \mu \mathrm{m}$. The detail of these reference SSDs are described 


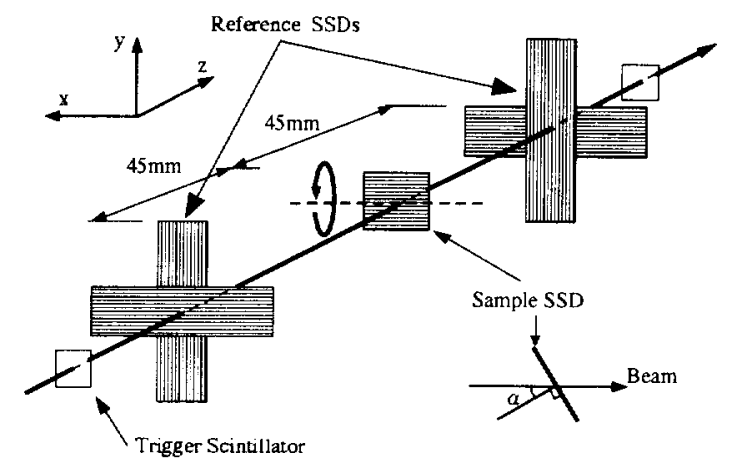

Fig. 2: The testbeam setup.

elsewhere[4].

\section{B. Detector}

For measurement of spatial resolution, we used sample SSDs fabricated by HAMAMATSU PHOTONICS K.K.. A sample SSD was mounted between the reference detectors, with the strips parallel to the $x$-axis(horizontal direction). This detector could be rotated about the local " $x$-axis" by a stepping motor system.

In this test, we used three SSDs whose strip pitches were 50, 125 and $250 \mu \mathrm{m}$, respectively. The parameters of these detectors are described in Table 1 . These detectors have an area of $10.0 \times 10.0 \mathrm{~mm}^{2}$. These SSDs are single-sided and have $\mathrm{n}^{+}$-strips on the ohmic side. P-stops are adopted between strips to separate the consecutive strips. The structure of these detectors is shown in Fig.3. The signals are readout with VA2 chips[5]. The bias voltage was $-70 \mathrm{~V}$ on the $\mathrm{p}$-side, while the $\mathrm{n}$-side was grounded.

Table 1

The parameter of sample SSDs.

\begin{tabular}{|l|r|r|r|}
\hline Sample & \multicolumn{1}{|c|}{ A } & \multicolumn{1}{c|}{ B } & \multicolumn{1}{c|}{ C } \\
\hline Strip pitch:p $[\mu \mathrm{m}]$ & 50 & 125 & 250 \\
\hline Number of strips & 200 & 80 & 40 \\
\hline Strip width: $\mathrm{Wn}[\mu \mathrm{m}]$ & 10 & 20 & 40 \\
\hline Electrode width: $\mathrm{Wr}[\mu \mathrm{m}]$ & 10 & 20 & 20 \\
\hline P-stop width:Wp $[\mu \mathrm{m}]$ & 30 & 95 & 200 \\
\hline Thickness $[\mu \mathrm{m}]$ & \multicolumn{4}{|c|}{300} \\
\hline Active area & \multicolumn{4}{|c|}{$20 \mathrm{M} \Omega$} \\
\hline Bias resistance & \multicolumn{3}{|c|}{$20 \mathrm{~mm}^{2}$} \\
\hline
\end{tabular}

The basic performance of these detectors was checked with a ${ }^{90} \mathrm{Sr} \beta$-source and IR laser[6] in advance of the testbeam experiment.

\section{Raw Data Processing}

For each event and each channel, the pulse-height and the noise are calculated with subtracting the offset and the common mode noise from ADC counts off-line[4].

Only if we find clusters on the whole set of four reference detectors, a track is reconstructed by interpolating hit positions of the upward and downward detectors. 

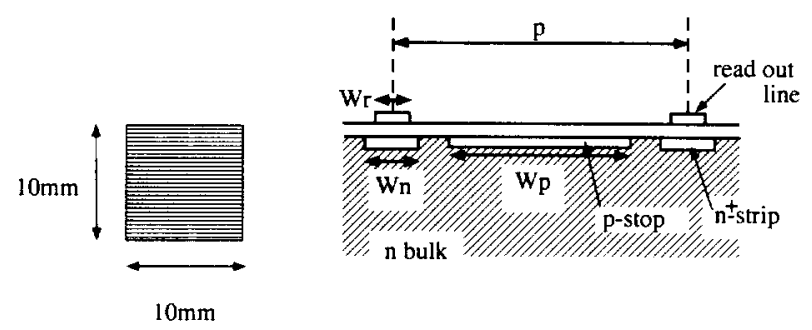

Fig. 3: Structure of sample SSD.

We calculate the hit position on the sample detector with the following steps;

(1) Data sparsification

(2) Cluster finding

(3) Delta ray cut

(4) Position calculation

The details of these algorithms are described below.

\section{A. Data sparsification}

At the read out system of BELLE-SVD, the sparse data scan will be adopted. In this analysis, therefore, we used the information of the strips which had larger charge than 3.7 times the noise level and its nearest neighbors only. The signals on the rest of strips were zero-suppressed.

\section{B. Cluster finding algorithm}

The charge created by a penetrating particle is collected by several consecutive strips, which form a cluster. In order to find the cluster, we first look for consecutive strips with the given width, whose sum of charges is higher than the given threshold. The threshold was defined as $5 \times \sqrt{\sum_{i} \sigma_{i}^{2}}$, where $\sigma_{i}$ is the r.m.s. noise of each strip. The cluster width, $N_{w}$, was defined as

$$
N_{w}=2+\operatorname{Int}\left(1.1 \times \frac{D}{p} \times \tan \alpha\right)
$$

where $D$ is the thickness of the silicon wafer $(300 \mu \mathrm{m}), p$ a pitch of the readout strip, $\alpha$ the incident angle, and Int $(x)$ the nearest integer to $x$. If there is more than one cluster, we accept a cluster which has the largest charge.

\section{Delta ray cut}

In order to reject the effect of $\delta$-rays, we applied the following algorithm as a $\delta$-ray cut for large incident angles more than $40^{\circ}$. When $Q_{i}$ is greater than $\langle Q\rangle+\sigma_{i}, Q_{i}$ is changed to $\langle Q\rangle=$ $\sum Q_{i} / N_{w}$.

\section{Position finding algorithm}

For computing the impact position of the particle passing through the detector, we used three different methods, i.e. (1) the analog centroid method(AC-method), (2) the analog head-tail method(AHT-method), (3) the non-linear method on $\eta$ (ETA-method) [7]. 


\section{1) Analog centroid method}

In the AC-method, the hit position, $X_{A C}$, is given by

$$
X_{A C}=\frac{\sum_{i \in \text { cluster }} x^{i} Q^{i}}{\sum_{i \in \text { cluster }} Q^{i}},
$$

where $x^{i}$ is each strip position defined at the center and $Q^{i}$ the charge collected on each strip. In the $\mathrm{AC}$-method, we used the information of the strips with larger charge than $\sigma_{i}$.

\section{2) Analog head-tail method}

For a large incident angle, the charge generated by a particle spreads among many strips. we assumed that the charge collected by strips was proportional to the path length of a particle in a $\mathrm{SSD}$. In this case, the charges collected by the central strips in the cluster do not contain position information. Therefore including this information (which is the case in the AC method) simply degrades the spatial resolution. In the AHT-method, we used the information from strips on both edges to solve this problem. We defined the head(tail) strip as a strip which had the smallest(largest) strip number and which had larger charge than three times noise level in a cluster. The hit position, $X_{A H T}$, is then given by

$$
X_{A H T}=\frac{x_{h}+x_{t}}{2}+\frac{Q_{t}-Q_{h}}{2 Q_{A V}} \times p
$$

where $x_{h}\left(x_{t}\right)$ is the position of the head(tail) strip and defined at the center of the strip. The charge collected on the head(tail) strip is $Q_{h}\left(Q_{t}\right)$. The average pulse height on the cluster is $Q_{A V}$.

3) ETA method

For a small incident angle, the charge generated by a particle is collected by almost a single strip. Fig.4(a) shows a simulation of the charge division between two consecutive strips. We define $\eta$ as

$$
\eta=\frac{Q_{\text {right }}}{Q_{\text {left }}+Q_{\text {right }}},
$$

where $Q_{\text {left }}\left(Q_{\text {right }}\right)$ is the charge collected on the left(right) side strip of the hitpoint.

In the ETA-method, the hit position, $X_{\eta}$, given by

$$
X_{\eta}=p f(\eta)+x_{l e f t}
$$

where $p$ is the readout pitch, $f(\eta)$ is an arbitrary growing function of $\eta$, with $f(0)=0$ and $f(1)=1$. We defined $f(\eta)$ as the cumulative probability distribution;

$$
f\left(\eta_{0}\right)=\frac{\int_{0}^{\eta_{0}} \frac{d N}{d \eta} d \eta}{\int_{0}^{1} \frac{d N}{d \eta} d \eta}
$$

where $d N / d \eta$ is the differential $\eta$ distribution. The function is obtained with fitting Fig.4(b) to the complex Gaussian. 


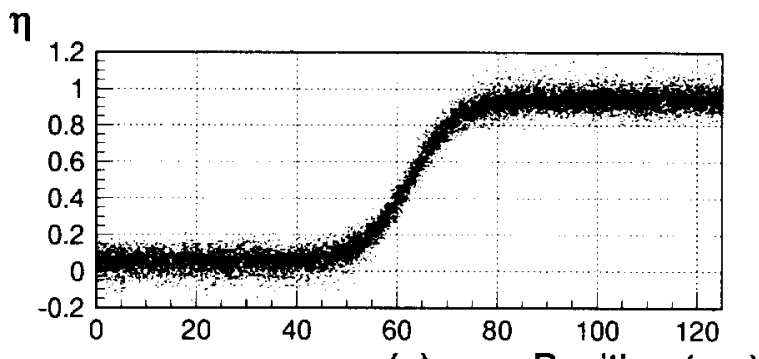

(a) Position $(\mu \mathrm{m})$

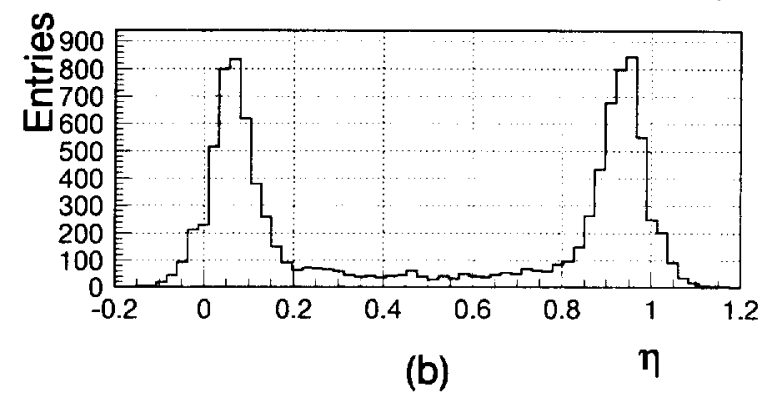

Fig. 4: Simulation of (a) Charge division and (b) $\eta$ distribution for the sample B(125 $\mu \mathrm{m})$.

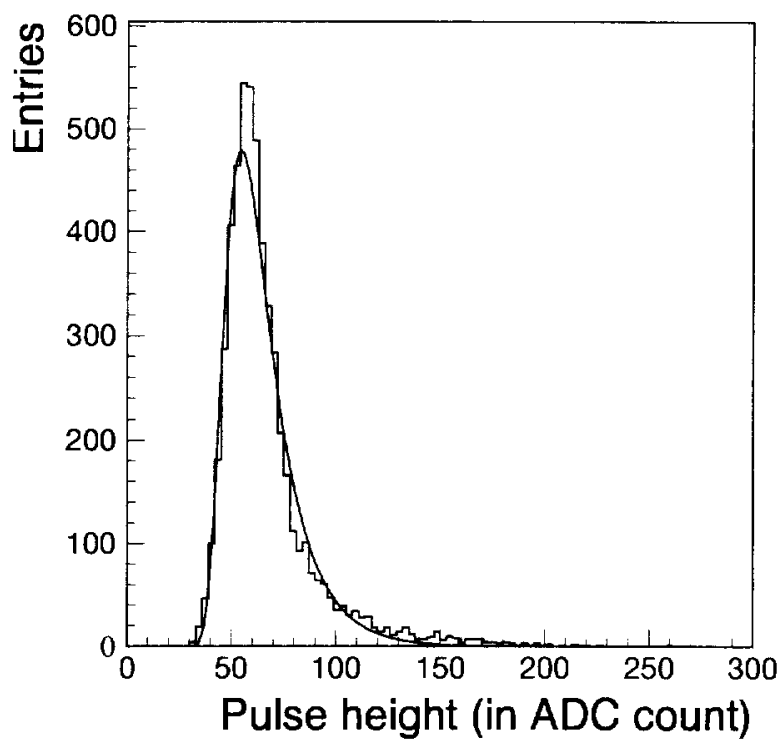

Fig. 5: Signal distribution with the sample $\mathrm{A}(50 \mu \mathrm{m})$.

\section{RESUlts}

\section{A. Signal distribution, $S / N$ ratio and Uniformity}

Fig. 5 shows a pulse-height distribution of the sample A with zero incident angle $\left(\alpha=0^{\circ}\right)$.

The $\mathrm{S} / \mathrm{N}$ ratio we obtained was $18.4 \pm 0.5$ for the sample $\mathrm{A}, 20.2 \pm 0.5$ for $\mathrm{B}$ and $16.8 \pm 0.5$ for $\mathrm{C}$, respectively.

Fig. 6 is a scatter plot of the pulse-height vs. the position of incidence obtained with the reference SSDs. In the figure the both ends (i.e. Position $=0,250 \mu \mathrm{m}$ ) correspond to the center of strips. It is seen that there is no sizable loss of charges between two strips. 


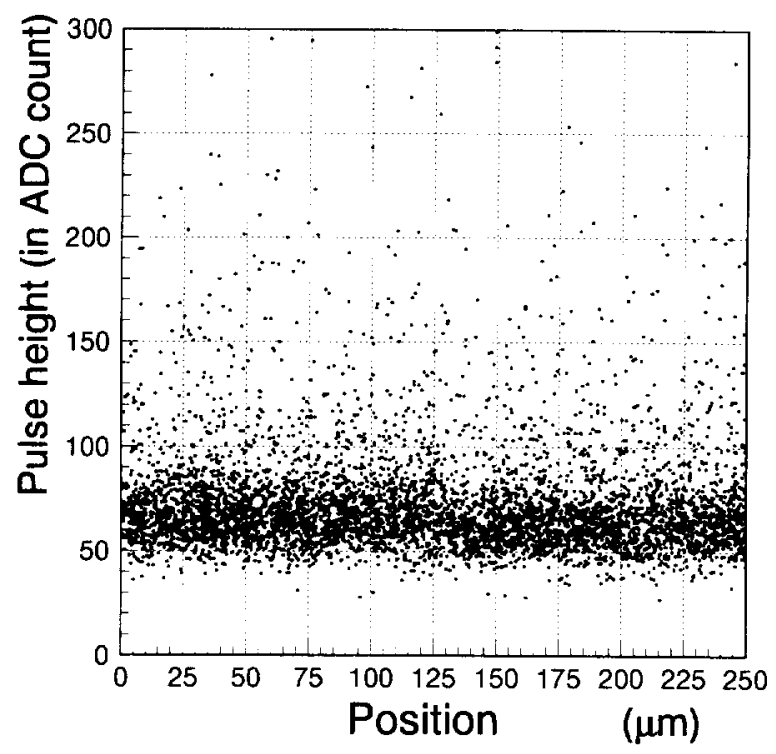

Fig. 6: The pulse-height distribution as a function of the position of incident particles.

\section{B. Residual distribution}

Fig. 7 shows the residual distributions of the sample $\mathrm{C}$ with the AC method. The differences between the point reconstructed by the reference telescope and the sample SSD is defined as residual.

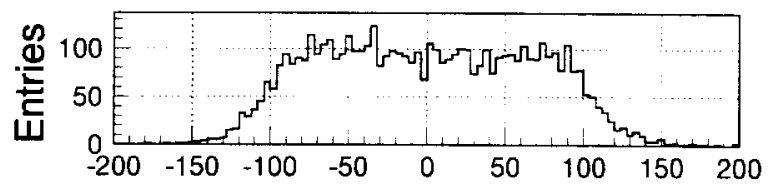

(a) Residual $(\mu \mathrm{m})$
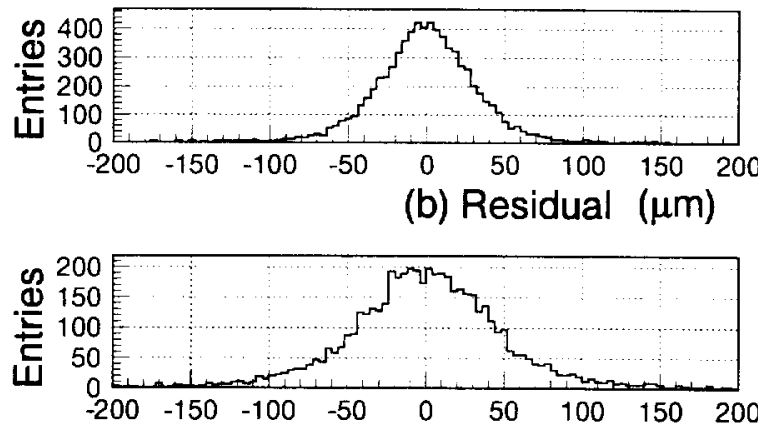

(c) Residual $(\mu \mathrm{m})$

Fig. 7: Residual distribution with the sample $\mathrm{C}(250 \mu \mathrm{m})$. (a) $\alpha=0^{\circ}$, (b) $\alpha=40^{\circ}$ and (c) $\alpha=60^{\circ}$.

We defined sigma $\left(\sigma_{f i t}\right)$ by fitting this distribution with the Gaussian distribution. This $\sigma_{f i t}$ contains the contributions from multiple Coulomb scattering and the finite resolution of the reference SSDs. These contributions need to be subtracted from $\sigma_{f i t}$. to obtain the intrinsic resolution of the sample SSD. Multiple scattering effect is calculated with taking the geometry of the setup and the momentum of the $\pi^{-}$beam into account [8]. Since there are SSDs for $y$-direction outside, this effect is larger in $y$-direction than that in $x$-direction. On the sample SSD, this effect is estimated to be $3.9 \pm 0.4 \mu \mathrm{m}$ for $x$-direction and $5.4 \pm 0.6 \mu \mathrm{m}$ for $y$-direction for particles with an incident angle of $0^{\circ}$. The resolution 
of each reference SSD has been measured by replacing the sample SSD with a reference SSD and we obtained $8.5 \pm 0.2 \mu \mathrm{m}$. On the sample SSD, this corresponds to an uncertainty of $6.0 \pm 0.1 \mu \mathrm{m}$.

\section{Spatial Resolution}

Data have been taken at ten different incident angles ranging from $0^{\circ}$ to $75^{\circ}$ for the sample $\mathrm{B}$ and $\mathrm{C}$ and at eleven different incident angles ranging from $0^{\circ}$ to $70^{\circ}$ for the sample $\mathrm{A}$. The spatial resolution of the sample SSDs as a function of the incident angle is shown in Fig.8-10 and Table 2-4. In the figure the open squares indicate data points with the $\mathrm{AC}$ method, the solid circles with the AHT method and the solid triangles with the ETA method, respectively. The solid lines are the simulation with the AC method. The detail of the simulation is described elsewhere[9]. With the AC method, measurements agree well with the simulations.

The AHT method was applied for samples B and C with large incident angles. It is seen that there is no sizable improvement once the sparsification is adopted. The peak of the signal read out from a single strip is expressed as $S / N \times \frac{p}{D \times \sin \alpha}$, which becomes smaller with the larger incident angle $(\alpha)$ and the smaller strip pitch $(p)$. When $p$ is $50 \mu \mathrm{m}$ (Sample A) and $\alpha$ is $75^{\circ}$, the expectation is 3.5 (with $\mathrm{S} / \mathrm{N}$ of 20 ) which is below the threshold of the sparsification. Therefore we did not apply the AHT method for the sample $A$.

With the ETA method, the resolution is sensitive to the $\mathrm{S} / \mathrm{N}$ ratio. We do not see a sizable improvement compared with the $\mathrm{AC}$ method in this test where the $\mathrm{S} / \mathrm{N}$ ratios are around 20 .

\section{Conclusion}

We measured the spatial resolution of silicon strip detectors for particles with incident angle ranging from $0^{\circ}$ to $75^{\circ}$. We tested three detectors which have $n^{+}$strips on the ohmic side with the pitch of 50,125 and $250 \mu \mathrm{m}$, respectively. With a large incident angle and a wide strip pitch, we obtained the results which agree well with simulation. This result proves that the intrinsic resolution of wide-pitch silicon strip detectors on the ohmic side is good enough to obtain the expected vertex resolution at the BELLE experiment. The position finding algorithm with the analog centroid method is effective enough for the strip pitches and the incident angles relevant for the BELLE experiment where $\mathrm{S} / \mathrm{N}$ ratio is around 20 and the sparsification is adopted for readout.

bibliography

\section{ACKNOWLEDGEMENTS}

We are grateful to the cooperation of the BELLE collaboration. We also thank the Physics department of KEK for their continuous encouragement.

Table 2

Summary of the spatial resolution on the sample A with the AC method.

\begin{tabular}{rcc|rcc}
\hline Angle & $\begin{array}{c}\sigma_{f i t} \\
{[\mathrm{deg}]}\end{array}$ & $\begin{array}{c}\text { Spatial } \\
\text { resolution } \\
{[\mu \mathrm{m}]}\end{array}$ & $\begin{array}{c}\text { Angle } \\
{[\mu \mathrm{m}]}\end{array}$ & $\begin{array}{c}\sigma_{f i t} \\
{[\mu \mathrm{m}]}\end{array}$ & $\begin{array}{c}\text { Spatial } \\
\text { resolution } \\
{[\mu \mathrm{m}]}\end{array}$ \\
\hline 0.0 & $16.3 \pm 0.2$ & $11.4 \pm 0.3$ & 29.4 & $20.4 \pm 0.3$ & $14.7 \pm 0.4$ \\
4.5 & $15.0 \pm 0.2$ & $9.3 \pm 0.4$ & 39.3 & $25.8 \pm 0.4$ & $19.7 \pm 0.5$ \\
9.3 & $13.9 \pm 0.2$ & $7.3 \pm 0.5$ & 49.3 & $36.2 \pm 0.8$ & $29.4 \pm 1.1$ \\
14.5 & $15.3 \pm 0.2$ & $9.3 \pm 0.4$ & 59.1 & $54.3 \pm 1.0$ & $45.3 \pm 1.2$ \\
19.3 & $16.4 \pm 0.2$ & $10.4 \pm 0.4$ & 69.2 & $79.6 \pm 1.3$ & $61.3 \pm 1.9$ \\
24.3 & $18.5 \pm 0.2$ & $12.9 \pm 0.4$ & & & \\
\hline
\end{tabular}


Table 3

Summary of the spatial resolution on the sample B with the AC method.

\begin{tabular}{|c|c|c|c|c|c|}
\hline Angle & $\begin{array}{c}\sigma_{f i t} \\
{[\mu \mathrm{m}]}\end{array}$ & $\begin{array}{l}\text { Spatial } \\
\text { resolution } \\
{[\mu \mathrm{m}]}\end{array}$ & $\begin{array}{l}\text { Angle } \\
{[\mathrm{deg} .]}\end{array}$ & $\begin{array}{c}\sigma_{f i t} \\
{[\mu \mathrm{m}]}\end{array}$ & $\begin{array}{l}\text { Spatial } \\
\text { resolution } \\
{[\mu \mathrm{m}]} \\
\end{array}$ \\
\hline 0.0 & $32.1 \pm 0.2$ & $29.9 \pm 0.3$ & 49.2 & $31.7 \pm 0.4$ & $23.6 \pm 0.6$ \\
\hline 9.3 & $25.9 \pm 0.2$ & $23.0 \pm 0.3$ & 59.2 & $39.0 \pm 0.5$ & $25.1 \pm 1.0$ \\
\hline 19.2 & $19.3 \pm 0.2$ & $14.6 \pm 0.4$ & 64.1 & $47.3 \pm 0.6$ & $28.6 \pm 1.3$ \\
\hline 29.2 & $22.4 \pm 0.3$ & $17.4 \pm 0.4$ & 69.2 & $60.1 \pm 0.8$ & $32.0 \pm 2.1$ \\
\hline 39.2 & $25.6 \pm 0.3$ & $19.5 \pm 0.5$ & 74.1 & $80.8 \pm 1.0$ & $30.0 \pm 4.4$ \\
\hline
\end{tabular}

Table 4

Summary of the spatial resolution on the sample $\mathrm{C}$ with the $\mathrm{AC}$ method.

\begin{tabular}{|c|c|c|c|c|c|}
\hline [deg.] & $\begin{array}{l}\sigma_{f i t} \\
{[\mu \mathrm{m}]}\end{array}$ & $\begin{array}{l}\text { Spatial } \\
\text { resolution } \\
{[\mu \mathrm{m}]}\end{array}$ & $\begin{array}{l}\text { Angle } \\
\text { [deg.] }\end{array}$ & $\begin{array}{c}\sigma_{f i t} \\
{[\mu \mathrm{m}]}\end{array}$ & $\begin{array}{l}\text { Spatial } \\
\text { resolution } \\
{[\mu \mathrm{m}]}\end{array}$ \\
\hline 0.0 & $67.2 \pm 0.7$ & $66.2 \pm 0.7$ & 49.2 & $37.7 \pm 0.5$ & $31.3 \pm 0.7$ \\
\hline 9.7 & $63.2 \pm 0.6$ & $62.1 \pm 0.6$ & 59.1 & $43.8 \pm 0.6$ & $32.0 \pm 1.0$ \\
\hline 18.9 & $48.1 \pm 0.4$ & $46.4 \pm 0.5$ & 64.2 & $54.2 \pm 0.7$ & $38.8 \pm 1.1$ \\
\hline 29.1 & $36.4 \pm 0.3$ & $33.6 \pm 0.4$ & 69.1 & $63.3 \pm 0.8$ & $37.9 \pm 1.9$ \\
\hline 39.2 & $29.8 \pm 0.3$ & $24.7 \pm 0.4$ & 74.1 & $83.4 \pm 1.7$ & $37.5 \pm 4.6$ \\
\hline
\end{tabular}

\section{REFERENCES}

[1] The BELLE Collaboration, BELLE Progress Report, KEK 96-1, 1996

[2] The BELLE Collaboration, A Study of CP Violation in B Meson Decays, Technical Design Report, January 1995

[3] For example, H. Pernegger, Dissertation, HEPHY Vienna (1996)

[4] H. Hanai et al, Nucl. Instr. and Meth. A314(1992), 455

[5] O. Toker et al, Nucl. Instr. and Meth. A340(1994), 572

[6] M. Hazumi et al, IEEE NSS 233-18, October 1995

[7] R. Turchetta Nucl. Instr. and Meth. A335(1993), 44

[8] G. R. Lynch Nucl. Instr. and Meth. B58(1991), 6

[9] BELLE Task Force Report, KEK Report 93-1, 1993. 


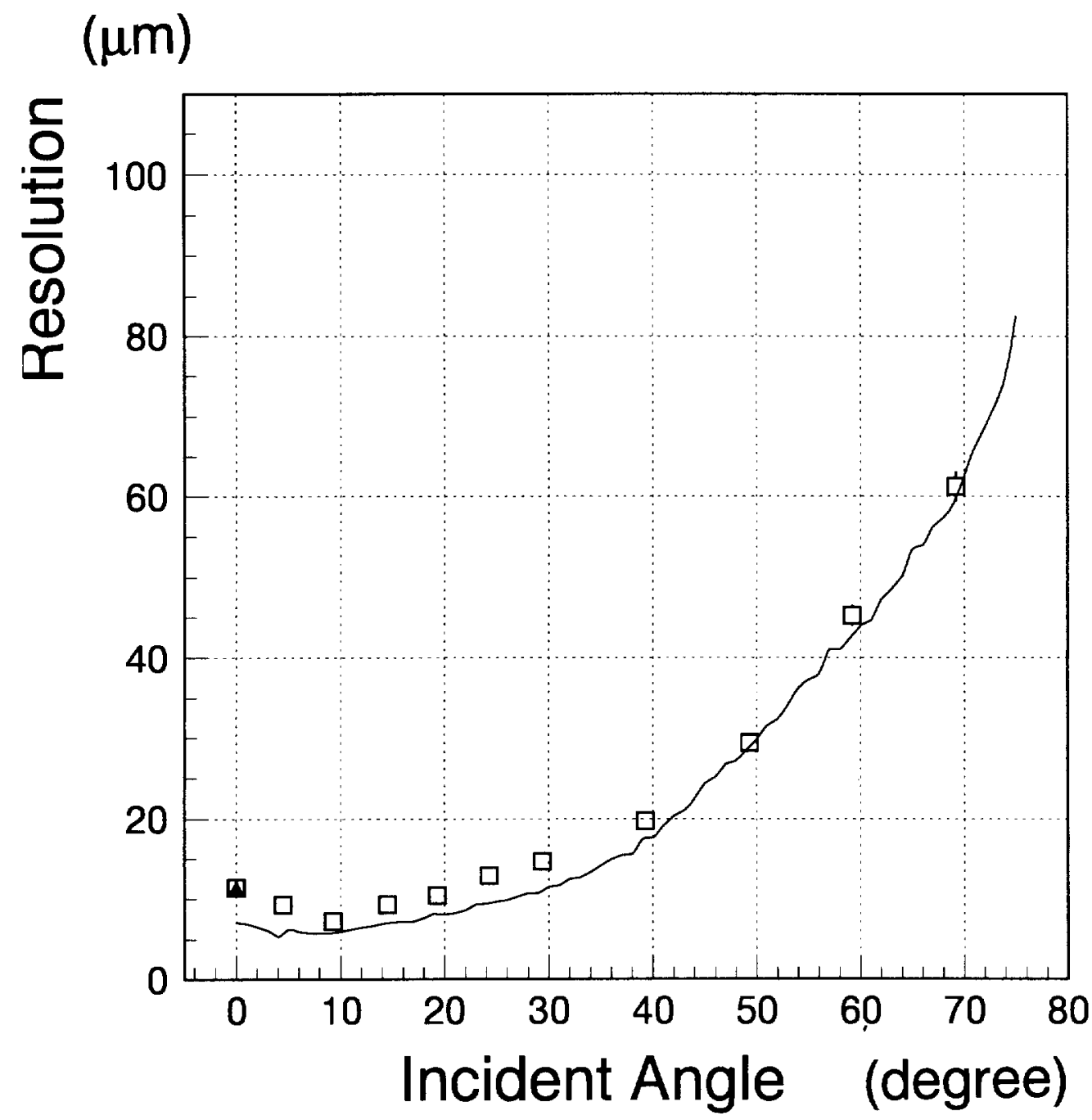

Fig. 8: Spatial resolution with sample $\mathrm{A}(50 \mu \mathrm{m})$. 


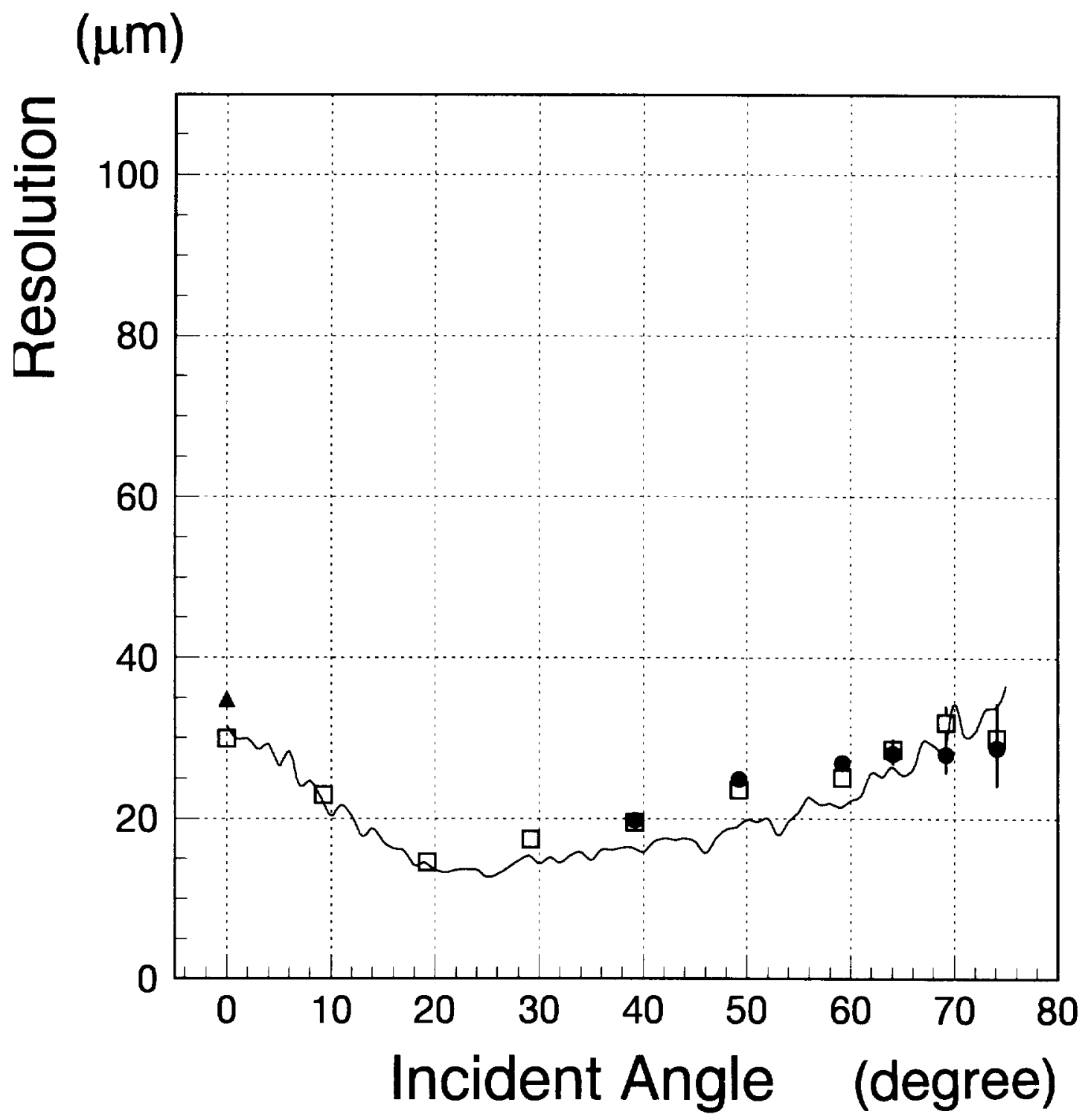

Fig. 9: Spatial resolution with sample $\mathrm{B}(125 \mu \mathrm{m})$. 


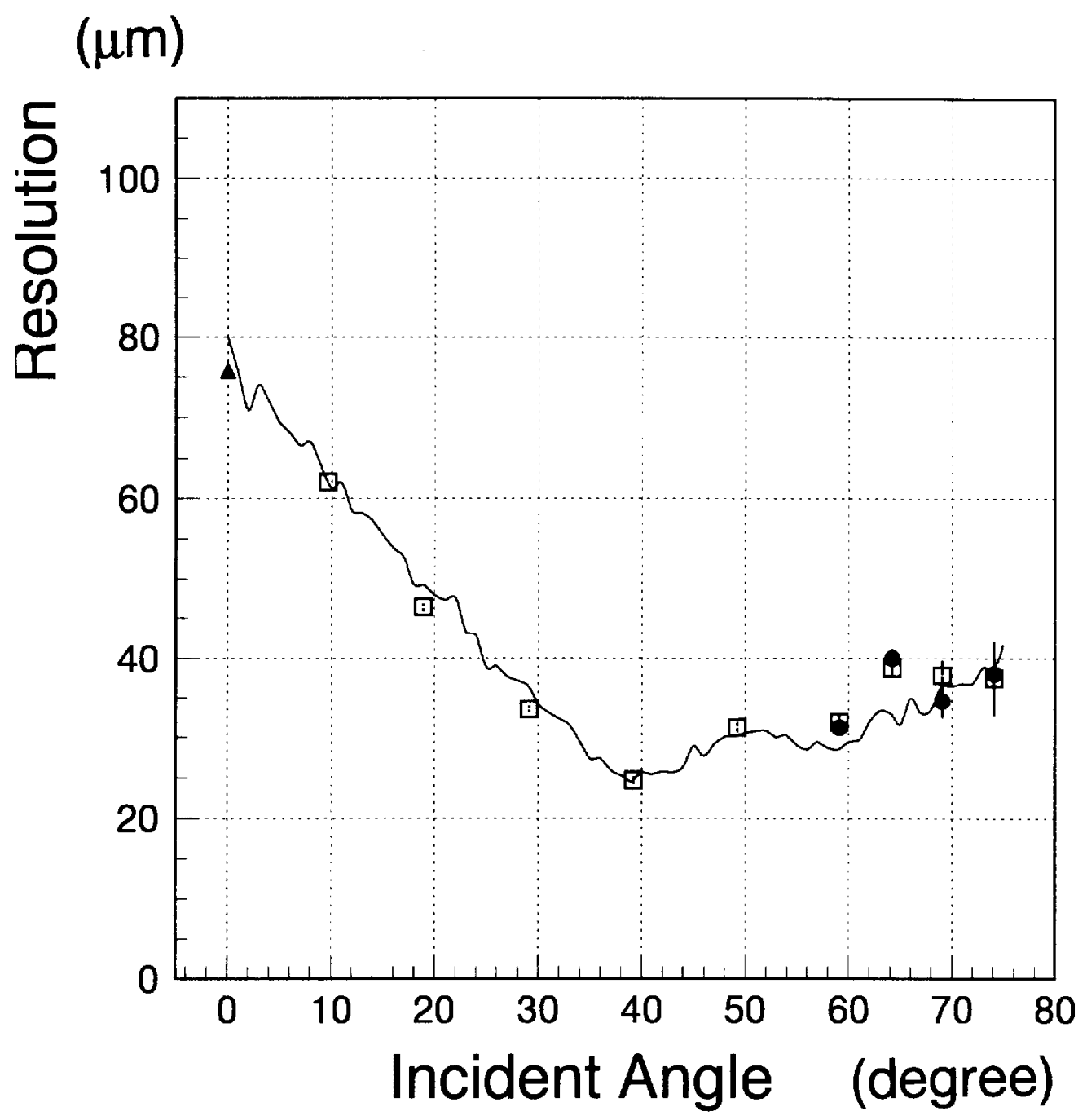

Fig. 10: Spatial resolution with sample $\mathrm{C}(250 \mu \mathrm{m})$. 